MAGNETOHYDRODYNAMICS Vol. 54 (2018), No. 1-2, pp. 163-166

DOI: $10.22364 / \mathrm{mhd} .54 .1-2.29$

\title{
ELECTRICAL CONDUCTIVITY OF INVERSE MAGNETIC FLUID EMULSION
}

\author{
A. Zakinyan \\ Department of General and Theoretical Physics, North-Caucasus Federal University \\ 1 Pushkin str., 355009 Stavropol, Russia \\ e-Mail: zakinyan.a.r@mail.ru
}

The paper deals with an experimental study of the emulsion whose dispersion medium is a magnetic fluid, whereas the disperse phase is formed by water. It is demonstrated that chain aggregates are formed from the disperse phase drops under the action of an external magnetic field. The change of the emulsion microstructure affects its electrical properties. The emulsion specific electrical conductivity was measured. It was found that under relatively weak external magnetic fields the emulsion electrical conductivity may change several times.

Introduction. Anisotropic heterogeneous matter constitutes an important field of basic research and applications. One of the approaches to produce materials which can become anisotropic under external action is based on the use of composite materials, whose properties strongly depend on their structural microgeometry. Recently many attempts have been undertaken to create new liquid magnetizable composites based on magnetic fluids that can more effectively interact with external magnetic fields than pure magnetic fluids [1-3]. The microstructure of such composites can be controlled by external magnetic fields. An example of the magnetic fluid composites is magnetic fluid emulsion. Magnetic emulsions are disperse systems consisting of two liquid phases; one of them is a magnetic fluid $[4,5]$. Some properties of magnetic fluid emulsions with dispersed droplets forming chain-like structures under the action of the magnetic field have been studied earlier $[6,7]$. The studies of magnetic emulsion macroscopic properties are of great interest due to the possibility of their efficient control by exposing to external force fields. It should be noted that emulsions with the disperse phase presented by a magnetic fluid have been studied mostly. In the context of electrical properties of the medium, it is of interest to study a magnetically controllable emulsion whose disperse phase has a pronounced electrical conductivity if compared to that of the dispersion medium. This lead to the investigation of invers magnetic fluid emulsions.

Previously, some results on the electrical properties of inverse magnetic fluid emulsions were presented in [8]. The emulsion of glycerin microdrops in a magnetic fluid was studied in [8]. The further development of the research is presented there. Particularly, it was expected that the use of water as a disperse phase material would lead to a more pronounced response of the electrical properties to the magnetic field action. In addition, the anisotropic character of the macroscopic properties should be studied in more details.

The present paper address the specific features of the macroscopic electrical conductivity of the magnetic emulsions caused by the formation of chain aggregates from the disperse phase drops under the effect of an external magnetic field. Such structure formation must lead to peculiar features of the conductivity of such media.

1. Materials and methods. As an object of the study, a magnetic emulsion was used whose dispersion medium is a magnetic fluid, whereas the disperse phase is formed by water. The emulsion was produced from a kerosene-based mag- 


\section{A. Zakinyan}

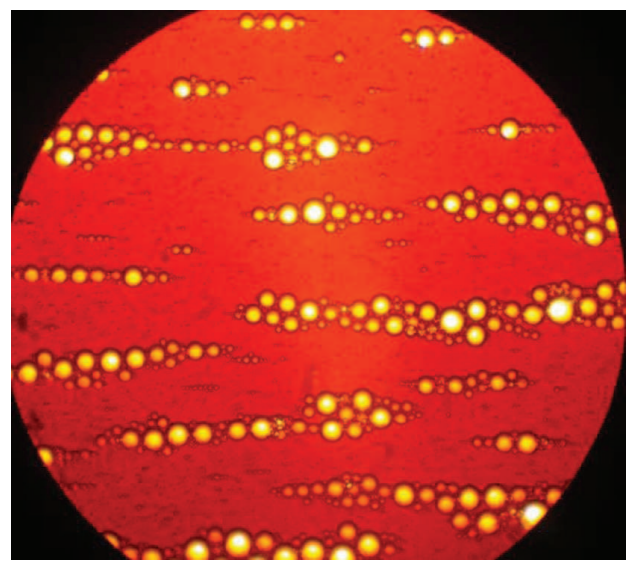

Fig. 1. Inverse magnetic emulsion with water as the disperse phase exposed to a uniform steady magnetic field directed horizontally.

netite magnetic fluid. The initial magnetic permeability of the magnetic fluid was $\mu \cong 1.5$ and the specific electrical conductivity was $\lambda_{\mathrm{e}} \cong 2 \cdot 10^{-6} \mathrm{~S} / \mathrm{m}$. Water was chosen as a disperse phase substance because it has a comparatively large specific electrical conductivity $\left(\lambda_{\mathrm{i}} \cong 4 \cdot 10^{-2} \mathrm{~S} / \mathrm{m}\right.$, in our case) if compared with a magnetic fluid dispersion medium. Such emulsion may be classified as "water-in-oil". Due to the great specific conductivity of the disperse phase compared with the dispersion medium, a significant change in macroscopic electrical properties of such emulsion during structure formation under the effect of external fields can be expected. The mean size of the emulsion disperse phase drops was $40 \mu \mathrm{m}$.

First, the structural properties of the water drops in the magnetic fluid synthesized emulsions exposed to an external magnetic field were studied. For this purpose, an emulsion specimen was subject to a steady uniform magnetic field. The structural transformations in the emulsion were observed using an optical microscope. The interaction of the induced magnetic moments of the droplets initiated the aggregation into chain structures aligned with the field intensity vector (Fig. 1).

The specific electrical conductivity $\lambda$ of the emulsion was studied using the bridge method. To determine the specific conductivity, the emulsion under study was placed into a rectangular cell. Then the cell active conductivity was measured. In the measurements, the $10 \mathrm{kHz}$ measurement signal was used. The specific conductivity $\lambda$ was calculated from the expression $\lambda=G / A$, where $G$ is the active conductivity of the emulsion-filled cell; $A$ is the constant of the cell predetermined by using a standard 0.01 aqueous solution of potassium chloride.

To study the effect of the magnetic field on the measured values, the emulsionfilled cell was exposed to the steady uniform magnetic field created by Helmholtz coils. The cell could rotate around a vertical axis, which allows varying the mutual orientation of the intensity vectors of the external magnetic and measuring electrical fields with respect to each other. The dependences of the specific electrical conductivity of the magnetic emulsion on the magnetic field strength and direction were studied.

2. Results. It has turned out that the electrical conductivity of the emulsions depends on the applied magnetic field strength and direction. Fig. 2 shows the dependences of the emulsion conductivity relative variation on the external magnetic field strength $H$ when the magnetic field is parallel and perpendicular to the measuring electrical field. It is seen that under the influence of the mag- 


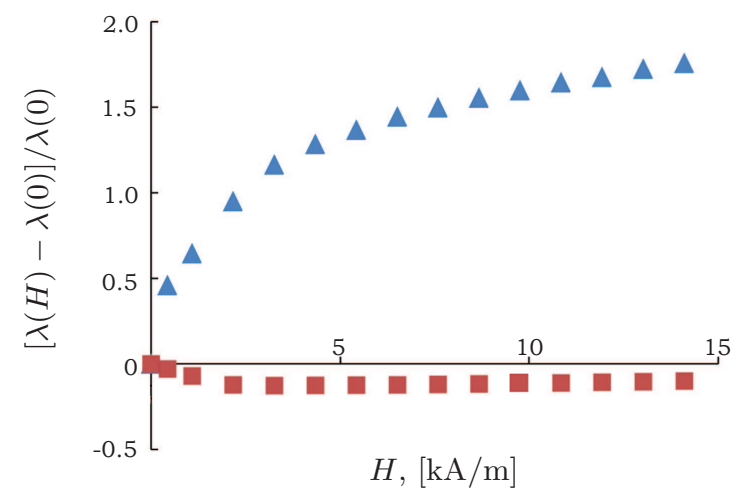

Fig. 2. Dependence of the relative variation of the electrical conductivity of magnetic fluid emulsion on the magnetic field strength. Triangles: magnetic field parallel to electrical field; squares: magnetic field perpendicular to electrical field. The emulsion volume concentration is 0.3 .

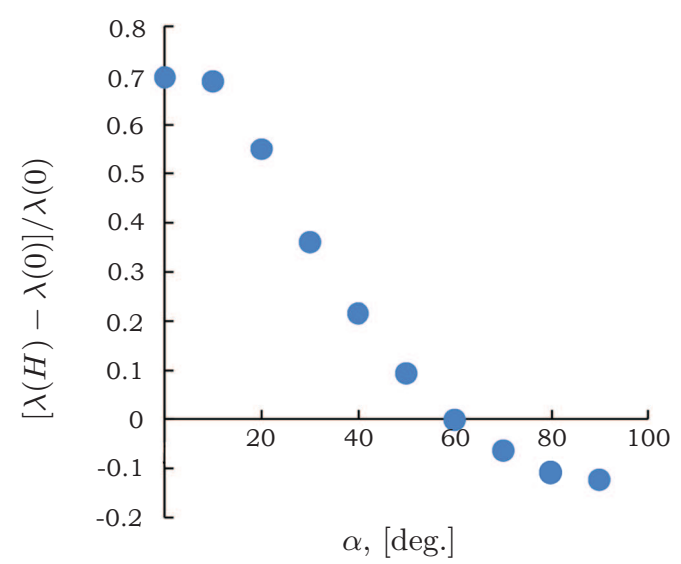

Fig. 3. Dependence of the relative variation of the specific electrical conductivity of magnetic fluid emulsion on the angle between the directions of electric and magnetic fields.

netic field whose direction coincides with that of the electrical measuring field, the conductivity increases if compared with the initial one. When the field direction is orthogonal, the emulsion conductivity shows a less pronounced decrease.

To reveal the anisotropic character of the macroscopic electrical properties of the magnetic fluid emulsion exposed to the magnetic field, the electrical conductivity of the emulsion was measured as a function of the angle between the directions of the external magnetic field and the electric measuring field (Fig. 3). The measurements were made for an emulsion with the volume concentration 0.2 and the external magnetic field strength $H=1.1 \mathrm{kA} / \mathrm{m}$.

3. Conclusions. So, in the presented work we have studied a new type of soft magnetic composites which is an inverse magnetic fluid emulsion with a chain-like microstructure of the disperse phase droplets. It has been demonstrated that the emulsion becomes anisotropic under the action of an external magnetic field. The anisotropic microgeometry of the emulsion leads to the anisotropy of the macroscopic parameters which become field-dependent. The emulsions studied can be categorized as a fluid with magnetic-field-controllable macroscopic electrical properties. 


\section{A. Zakinyan}

Acknowledgements. This work was supported by the grant of the President of the Russian Federation No. MK-3169.2017.2, by the grant of the Russian Foundation for Basic Researches (project No. 18-03-00279) and also by the Ministry of Education and Science of the Russian Federation in the framework of the governmental order for scientific research works (project No. 3.5822.2017/8.9).

\section{References}

[1] J. Popplewell, R.E. Rosensweig. Magnetorheological fluid composites. J. Phys. D: Appl. Phys., vol. 29 (1996), pp. 2297-2303.

[2] M.T. Lopez-Lopez，P. Kuzhir，S. Lacis， G. Bossis， F. Gonzalez-CABallero, J.D.G. Duran. Magnetorheology for suspensions of solid particles dispersed in ferrofluids. J. Phys.: Condens. Matter., vol. 18 (2006), S2803S2813.

[3] G. Helgesen, E. Svasand, A.T. SkJeltorp. Nanoparticle induced selfassembly. J. Phys.: Condens. Matter., vol. 20 (2008), 204127.

[4] A. Zakinyan, Y. Dikansky. Drops deformation and magnetic permeability of a ferrofluid emulsion. Colloids Surf. A, vol. 380 (2011), pp. 314-318.

[5] A.O. Ivanov, O.B. Kuznetsova. Nonmonotonic field-dependent magnetic permeability of a paramagnetic ferrofluid emulsion. Phys. Rev. E, vol. 85 (2012), 041405.

[6] S. Brojabasi, B.B. Lahiri, J. Philip. External magnetic field dependent light transmission and scattered speckle pattern in a magnetically polarizable oil-in-water nanoemulsion. Physica B, vol. 454 (2014), pp. 272-278.

[7] F. Montagne, S. Braconnot, O. Mondain-Monval, C. Pichot, A. Elä̈ssaRI. Colloidal and physicochemical characterization of highly magnetic O/W magnetic emulsions. J. Dispersion Sci. Technol., vol. 24 (2003), pp. 821-832.

[8] A. Zakinyan, Y. Dikansky, M. Bedzhanyan. Electrical properties of chain microstructure magnetic emulsions in magnetic field. J. Dispersion Sci. Technol., vol. 35 (2014), pp. 111-119. 